\title{
La participación de profesionales en orientación en la gestión de la motivación en las organizaciones educativas
}

The professional counselor's participation for managing the motivation in the educational organizations

Recibido 21 octubre 2012 • Aceptado 23 noviembre 2012 • Corregido 26 noviembre 2012

\author{
Wilbert Porras Quirós ${ }^{1}$ \\ Universidad Nacional \\ San José, Costa Rica \\ willrony@yahoo.es
}

Resumen. Los cambios en la sociedad, así como en los mercados laborales, han generado una variación en la visión de la persona empleada dentro de las organizaciones existentes, resaltándose la importancia de que estas se mantengan motivadas, lo cual genera mayor calidad en los productos y servicios producidos. Además, se rescata la contribución que puede brindar el trabajo de profesionales en Orientación en consolidar un adecuado ambiente organizacional, que estimule el rendimiento de las y los colaboradores de la organización, mediante el fortalecimiento de estrategias que aumenten la motivación de cada uno de ellos y ellas.

Palabras claves. Motivación, Orientación, organizaciones educativas, clima laboral, personas colaboradoras.

Abstract. Changes in society and in different labor markets have generated a change in the vision of the person employed within the various existing organizations. This demonstrates the importance for the people been motivated, which generates higher quality products and services. In addition, it is relevant to mention the contribution that may provide the counselor's labor in the consolidation of an appropriate organizational environment. Further, this encourages the collaborators to have a better performance by the strengthening of strategies that stimulate and increase the motivation of each of them.

Keywords. Motivation, counseling, educational organizations, work environment, collaborators.

Bachiller en Orientación en la Universidad Nacional de Costa Rica. Experiencia en trabajo con adolescentes, madres, padres de familia y pescadores que viven en zonas rurales-costeras (Isla Venado, Isla Caballo y Cabuya) como asistente en el Área de Desarrollo Humano del Programa de Desarrollo Integral Comunitario Costero de la Universidad Nacional. Facilitador de cursos participativos en la Universidad Nacional. 


\section{Introducción}

La educación es un proceso dinámico, el cual contempla al ser humano como alguien integral, es decir "(...) puede entenderse desde diversos sentidos y formas, según las épocas y las disciplinas que se encargan de estudiarlo" (Solórzano, 2008, p. 349). Por su parte, las organizaciones educativas están conformadas por el personal docente, administrativo y el estudiantado, en general. Las dos primeras agrupaciones mencionadas, quizás son quienes tienen una mayor responsabilidad en desarrollar óptimos procesos de enseñanza aprendizaje, en donde se mantenga como eje transversal de trabajo la motivación de sí mismos y de los individuos a cargo.

Aunado a lo anterior, para mantener en el personal docente y administrativo una motivación adecuada, es necesario contemplar aspectos relacionados con el clima organizacional, definido por Quintero, Africano y Faría (2008) como“(...) un componente multidimensional de elementos que pueden descomponerse en términos de estructuras organizacionales, tamaño de la organización, modos de comunicación, estilos de liderazgo de la dirección, entre otros" (p. 35).

Ante el panorama expuesto previamente, se contempla como objetivo del presente escrito, la necesidad de analizar y dar a conocer la importancia de la motivación dentro del clima organizacional de las instituciones educativas, abordado desde la visión de la disciplina de la orientación como estrategia para lograr calidad laboral y de vida en las personas implicadas. Se busca evidenciar los aportes que pueden brindar los profesionales en orientación -en conjunto con la dirección institucional- en la generación de procesos capaces de contribuir en la motivación de quienes están involucrados en el sistema educativo.

Es importante resaltar, que la orientación, según el Plan de Estudios de la División de Educación para el Trabajo de la Universidad Nacional de Costa Rica (2012) "es una carrera que prepara profesionales promotores del desarrollo integral de las personas, mediante procesos de desarrollo personal y vocacional. Estimula las relaciones interpersonales en la familia y en el ambiente laboral y social, para la construcción de una sociedad con mejor calidad de vida" (Introducción, párr. 1). Aunado a lo anterior, estos profesionales, con previa negociación con la dirección institucional, pueden desarrollar espacios de introspección personal y profesional en donde se construyan bases sólidas para un adecuado funcionamiento de la organización a partir de los aportes de las personas colaboradoras, contemplando aspectos de su integralidad (física, emocional, espiritual, social y cognitiva), con el fin de generar y mantener niveles adecuados de motivación en todos sus quehaceres mediante la propiciación del autocuidado, la expresión de emociones y sentimientos, entre otros.

Se rescata que la motivación es una fuerza motora capaz de permitirle al ser humano actuar eficazmente en los diferentes campos y actividades en donde se desarrolle. Investigaciones sobre el 
clima organizacional, han logrado evidenciar que es trascendental trabajarla, pues a partir de ella, las personas colaboradoras (orientadas) puedan sentirse satisfechas con el papel adquirido dentro de la organización en la cual laboran (Barquero, 2005), así como en su vida en general, pudiendo de esta manera optimizar su rendimiento personal y laboral, convirtiéndose en un eje de acción importante para trabajar, a su vez, en la prevención de problemáticas del sistema educativo en general, como son las constantes incapacidades, conflictos interpersonales e incluso, la deserción y repitencia estudiantil; campo el cual la administración de la educación asume un papel relevante.

Entre las personas que han trabajado desde lo conceptual la motivación, se encuentra Barrionuevo (2001), quien la define cómo “(...) aquello que mueve, que empuja, lo que impulsa a la acción" (p. 15), continúa aportando que un equipo motivado se "(...) pone la camiseta a favor de la consecución de sus objetivos, lucha constantemente por conseguir sus metas, reconoce que existen obstáculos, sin embargo lucha por sobrepasarlos" (p. 15).

Es innegable destacar cómo en el Sistema Educativo Costarricense desde la gestión, es importante el fortalecimiento de los equipos de trabajo como herramienta para lograr conseguir altos estándares de efectividad dentro del quehacer del educador y educadora, posibilitando en ellos y ellas, espacios catárticos en donde los miembros puedan recibir ayuda y apoyo mutuamente partiendo de la vivencia de situaciones similares, en las cuales las experiencias formativas y de vida se contemplen como un aspecto sobresaliente en la visualización de alternativas para conllevar exitosamente los retos y desafíos tenidos, propiciando la motivación personal a partir del apoyo grupal.

En estos equipos de trabajo, los profesionales en orientación pueden contribuir activamente en la promoción de procesos de toma de decisiones, de comunicación y de introspección grupal y personal con los cuales se fortalezca la dinámica grupal como eje primordial para mantener niveles adecuados de motivación.

\section{La motivación en las organizaciones educativas}

Cabe destacar que la motivación está influenciada por factores: primeramente extrínsecos, conformados por cualquier tipo de motivo ajeno a la persona que realiza la acción, no pueden ser controlados por ella como tal, responden a situaciones económicas o sociales a nivel macro; en segundo lugar, están los intrínsecos, corresponden a motivos o excusas internas generadas por la persona que realiza la acción, pueden ser manifestadas mediante conductas conscientes o inconscientes y finalmente, se encuentran los trascendentes, estos conciernen a un "efecto dominó", en donde la acción de una persona motivada se transmite a otra (Gómez y Pin, 1994). Esto hace evidente, cómo se pueden realizar diferentes tipos de intervenciones en las personas, cuya finalidad sea fortalecer su motivación. 
Si bien es cierto, los factores externos, internos y trascendentes, afectan positiva o negativamente la motivación de las personas, sin poder tener control directo sobre ellos, es fundamental su reconocimiento -y sobre todo- concienciarlos en los individuos, pues se pueden desarrollar las competencias y herramientas necesarias para asumir la responsabilidad correspondiente de la forma de reaccionar tenida ante ellos y de esta manera contribuir al desarrollo de agentes motivadores personales y de la organización educativa como tal.

Los factores externos, también se vinculan con conductas externas de las personas, reforzadas por la sociedad como lo es el salario y los halagos recibidos por los integrantes del grupo. Los factores internos, se relacionan con aquellas actividades que realiza el individuo para satisfacer intereses, curiosidades, necesidades y reacciones personales internas (Reeve, 1994). Estos elementos, al ser trabajados recíprocamente entre la dirección y el profesional en orientación, atañe a ver como los individuos son parte de un todo, en donde sus conductas o reacciones pueden ser modificadas o mantenidas mediante el apoyo que se les brinde.

Los profesionales en orientación tienen dentro de sus retos de trabajo -junto con el director o directora de la organización educativa- unificar e interiorizar la visión y misión institucional entre sus miembros, con la finalidad de crear propuestas pertinentemente efectivas para mejorar las condiciones y oportunidades del personal, esto fortalece a su vez, la vinculación y compromiso de sus miembros con la entidad (Barquero, 2005), llegando a transformarse en garantías viables para mantener la motivación y el interés por el trabajo continuo.

Un estudio realizado por Condorí (2003) manifiesta lo siguiente: “(...) la "motivación es algo intrínseco del ser humano", las personas la poseen en prácticamente todas las ocasiones de la vida y en los diferentes espacios en los que se encuentren (social, familiar, académico, laboral....)" (p. 2). Como hace evidente este autor, el ser humano tiene la motivación como un eje propulsor que le permite actuar, y aunque proviene desde la parte interior del individuo (viéndose influenciado por factores externos como premios y castigos), también se puede estimular y reforzar con las condiciones que el medio le ofrece a éste; así como el apoyo dado por profesionales de ayuda, como son los orientadores y orientadoras de la educación.

\section{Estrategias de atención para la motivación en las organizaciones educativas.}

La motivación también se vincula como un factor emocional básico para el ser humano en sus diferentes contextos (personal, laboral y familiar entre otros), cuando se tiene, las tareas cotidianas se realizan con pasión, liberación y sin esfuerzo adicional, se ve en el trabajo un refugio a las frustraciones personales y laborales, aumenta la autoestima, el reconocimiento profesional y social (Espada, 2006, citado por Ramírez, Abreu y Badii, 2008). Por ende, surge la necesidad de darle la importancia respectiva a la temática y trabajar constantemente por 
fortalecerla, mediante la implementación de estrategias que refuercen factores internos y externos de las personas dentro de las organizaciones educativas con la finalidad de aumentar su espíritu positivista y afán de desarrollo.

En las instituciones educativas, es necesario el trabajo conjunto entre todas las personas que la conforman, pues "(...) persona y organización se encuentran en una constante interacción, que se fundamenta en una expectativa recíproca entre individuo y organización respecto del desempeño esperado y la retribución que se recibirá a cambio" (Ramírez et ál., 2008, p. 143). Ante ello, el profesional en orientación puede articular esfuerzos y estrategias, desde sus funciones y formación para lograrlo; sin embargo, muchas veces la actitud y forma de reacción de los individuos es algo que solo depende de sí mismos, ante esto, los esfuerzos deben ser conjuntos, y sobre todo, muy visionarios pues van en función de tener calidad de vida y excelencia profesional para contribuir con el quehacer de cada quien, en la optimización de los procesos de enseñanza aprendizaje.

Con el apoyo de la gestión del centro educativo, el profesional en orientación, al ser un especialista en el desarrollo de estrategias preventivas y de procesos de desarrollo humano (División de Educación para el Trabajo, 2012), puede, mediante ellas, dar atención integral individual y colectiva a las personas vinculadas a la institución educativa, buscando con ello, promover una motivación constante y adecuada dentro del personal docente y administrativo de la institución, mediante el fortalecimiento de:

- La comunicación asertiva: expresar lo que se siente y cree de forma clara y respetuosa (Aguilar y Vargas, 2010).

- Autoestima: amor por sí mismo, nace a partir del autoconocimiento (Arce, 2000).

- Empatía:capacidad de entender y posicionarse en las vivencias de otros, entendimiento, situarse en los zapatos de la otra persona (Sánchez, Oliva y Parra, 2006).

- Orientación vocacional: el desarrollo de la persona, y la prevención de problemas de toda índole; se interviene a través de programas (Bisquerra, 1990).

- Proyecto de vida: hace referencia a las aspiraciones hacia el futuro tenidas por las personas, lo cual, hace que se orienten los esfuerzos por obtener un nivel y una calidad de vida en donde se potencie el desarrollo personal (Chacón y Villalobos, 2005). Lo que responde a ¿qué es lo que quiero para mi futuro?, ¿qué hago? y ¿qué quiero hacer?

Estas estrategias es necesario completarlas con espacios para el manejo de estrés, recreación, ocio, desarrollo de habilidades y competencias sociales-personales, estilos de 
liderazgo, introspección y encuentro consigo mismo, entre otras, pues se debe ver al ser humano desde su integralidad, pues la motivación surge a partir del estado de satisfacción tenida en todas las áreas del individuo, y esto enriquece la calidez de la organización educativa.

\section{Gestión de la motivación en las organizaciones educativas}

La motivación a nivel colectivo se puede visualizar como fundamental, y es tanto así, que en los equipos de trabajo constituye un tema relevante, le facilita o impide avanzar con la tarea propuesta y por ende, con la consecución del o los objetivos de la organización educativa. En consecuencia, los modelos jerárquicos de las empresas exitosas se han transformado; ahora, se busca disminuir las jerarquías y empoderar a las personas colaboradoras para que se sientan con la capacidad de participar activamente en la generación de ideas productivas y pertinentes a la resolución de conflictos propios del medio y de las crecientes demandas de la sociedad moderna en sus lugares de trabajo (Barquero, 2005), perfil ideal de la persona educadora, quien a diario se encuentra con múltiples retos, en donde es importante que cuente con el apoyo y respaldo en las decisiones que debe tomar, por parte de profesionales especialistas en el área, así como de la dirección administrativa.

En la actualidad, se ha evolucionado en la visión de ver a las personas como simples empleados, cuya única motivación era recibir un salario, a verles como colaboradores activos, quienes con su quehacer favorecen a la institución y en el proceso, se autorrealizan como seres integrales. Este aspecto es fundamental de reforzar constantemente en los profesores y profesoras, quienes tienen una labor muy extenuante, en donde necesitan de espacios para hacer catarsis, al lado de profesionales expertos en la materia como son los profesionales en orientación.

Es importante hacer la salvedad de que en el presente escrito se utiliza el término equipo para hacer referencia a las personas que contribuyen con su trabajo en las organizaciones, pues esta es una de las maneras más adecuadas de realizar trabajo conjunto, caracterizándose principalmente porque todas las partes participan activamente dentro de los procesos desarrollados en la institución y con sus aportes enriquecen la formulación de alternativas para optimizar el rendimiento y la calidad de lo realizado en las organizaciones educativas (Barrionuevo, 2001).

La motivación en los equipos u organizaciones es dinámica; está en constante transformación debido a las características propias del contexto y las reestructuraciones que con el transcurso del tiempo y las demandas del mercado se produzcan. Ante este panorama, el profesional en orientación especializado en esta temática, mediante la preparación complementaria en estos tópicos o reforzando su formación en áreas afines, debe estar en una 
constante lectura de las situaciones emergentes, los cambios de la organización educativa, las necesidades y características de las personas colaboradoras para replantear o reforzar modelos de intervención acordes a las nuevas dinámicas presentadas en la institución en la cual se labore; trabajo que debe ser propiciado por adecuados procesos de gestión en las organizaciones educativas.

Es necesario estar en un proceso constante de vinculación con la institución educativa, con el fin de conocerla a profundidad y trabajar activamente en la construcción de procesos estratégicos para la prevención de la resistencia al cambio que posiblemente se podría producir en las diferentes etapas de evolución vividas en la actualidad, producto de los aportes generados por la ciencia, la tecnología, las exigencias de la sociedad moderna, y los procesos de reubicación dentro del entorno laboral que pueden disminuir la motivación.

Para Abarca (1995), “(...) la motivación, es la que orienta las acciones, define por qué hacemos las cosas y hacia dónde nos dirigimos" (p. 5). En las organizaciones educativas, la motivación se establece principalmente por la forma en cómo se perciben las situaciones ocurridas en su acontecer, es por ello que un determinado evento puede ser el detonante para que el equipo se active y avance o por lo contrario, deje de ser funcional, según la interpretación dada por sus integrantes, así como el apoyo de profesionales competentes para tal fin.

El rol del profesional en orientación, en este sentido, es de ser un mediador y clarificar de los eventos acontecidos en el contexto inmediato de las personas, posibilitando la identificación de las principales emociones y sentimientos generados, con la finalidad de propiciar la compenetración necesaria para el surgimiento de la motivación. Es común escuchar en la cotidianidad, que aquella persona logró alcanzar su meta por su motivación, esta situación en forma similar funciona en las organizaciones educativas, en la medida que desde la gestión institucional se logren mantener motivados sus colaboradores van a lograr a menor plazo y con mayor calidad alcanzar las metas propuestas.

Algunos de los beneficios de trabajar con personas motivadas es que se puede alcanzar mayor bienestar integral, tener una visión más clara de la tarea a realizar, hay mayor energía, se actúa desde el "Yo", o sea "el aquí y el ahora", se ven beneficiadas las relaciones intra e interpersonales, generándose más satisfacción en las necesidades personales y laborales.

Cabe resaltar, la importancia que tiene fomentar las relaciones interpersonales en las organizaciones educativas ante lo cual la administración es estratégica, de manera que se disminuya la individualidad, pues esto tiene influencia decisiva en el comportamiento del colectivo (Yañez, Arenas y Ripoll, 2010). Si las personas logran sentir empatía por las otras, 
un sentido de pertenencia y respeto se construyen bases más sólidas para el intercambio de ideas, apoyo y confianza entre todas y todos, disminuyendo la rivalidad y aumentando la sana competencia. Para abordar lo mencionado anteriormente, el o la profesional de orientación puede informar, capacitar y asesorar acerca del adecuado desarrollo de procesos de comunicación asertiva como una herramienta ideal para expresar lo que se piensa y siente de manera adecuada y pertinente, pensando en el bienestar propio y de la otra parte, todo esto es posible con la generación de espacios organizacionales que la gestión desde sus competencias puede propiciar.

El orientador y orientadora que labora en organizaciones educativas públicas o privadas, es necesario que se empodere, se posicione en su quehacer y busque crear una cultura organizacional de colaboradores y colaboradoras con capacidad de tener voz y voto en la toma de decisiones trascendentales, con un alto nivel de escucha activa y competencias de negociación, con habilidades para defender sus ideales y apertura al reconocimiento de la contraparte, de tal manera que sientan confianza en participar de los procesos de la institución activamente y no caigan en una profunda frustración, si en algún momento sus propuestas son enriquecidas con los aportes de otras personas y se transforman los preceptos aportados inicialmente.

Las diferencias entre las y los integrantes de las organizaciones educativas, al no ser elaboradas se convierte en una serie de amenazas, que al evidenciarse, se transforman en detonante para que la dinámica organizacional se vea afectada, surja la desmotivación y se dé un estancamiento en la espiral de crecimiento de la institución, surgiendo fantasías e ideas irracionales, las cuales inhabilitan al equipo y lo convierten en disfuncional (Yañez et al., 2010). Ante esta panorámica, el profesional en orientación puede considerar la motivación como el eje impulsor de cambio, buscando confrontar adecuadamente la situación con el apoyo de la gestión del centro educativo, y permitiendo actuar al equipo, no aislándolo sino promoviendo la visualización de los roles que se están ejerciendo y cómo estos afectan o ayudan a la dinámica tenida.

Recabando más preceptos acerca de la motivación humana, aparece indiscutiblemente McClelland, citado por Alles (2011), quien la basa en tres sistemas que gobiernan el comportamiento humano, los cuales se describen de la siguiente manera:

- Los logros como motivación: las personas que utilizan este tipo de motivación actúan donde se ve la posibilidad de marcar una diferencia sustancial, no trabaja en situaciones donde no haya posibilidades de mejora. Este tipo de personas (con alta motivación al logro) prefieren tener responsabilidad personal por los resultados. 
- El poder como motivación: este tipo de motivación se basa en las fuerzas que se posean, es competitivo, este tipo de personas han tenido dificultad para adecuarse a las nuevas tendencias del mercado laboral.

- La pertenencia como motivación: se cree que se desarrolla de la necesidad de estar con otras personas, de la vinculación en sociedad de los individuos. (p. 48)

Como se evidencia, el o la profesional de orientación es necesario que analice cuáles son los sistemas de motivación presentados dentro de la institución educativa y trabaje en ellos -en coordinación con el director o directora-, ya sea para seguir reforzándolos o para generar nuevos esquemas de trabajo e intervención, lo cuales produzcan el desarrollo de competencias personales y laborales que posibiliten la optimización de los recursos utilizados en el mejoramiento de estrategias organizacionales, las cuales permitan un mejor desempeño, aplicación de técnicas y estrategias motivadoras.

De esta forma, McClelland, mencionado por Alles (2011) explica que para mejorar la motivación humana se deben seguir las siguientes recomendaciones:

Tener en cuenta tanto las necesidades de la organización como de las personas, de modo de lograr satisfacerlas. Para ello se deberá tener un sistema descriptivo de puestos y modelo de competencia actualizado y adecuado a las necesidades de la organización y, a continuación una correcta evaluación de las capacidades de las personas que ocupan esos puestos.

Implementar premios y castigos mediante un adecuado sistema de evaluación del desempeño que permita valorar y premiar la gestión de las personas. (p. 49)

Desde este enfoque teórico, el o la profesional de orientación puede sustentar su trabajo en el abordaje de las necesidades e intereses de las persona colaboradoras de la organización educativa, para que puedan acceder a condiciones básicas de trabajo que les permitan sentirse cómodas en su puesto y contribuyan a dar un mayor rendimiento en él.

Además, otro de los ejes medulares, en donde puede contribuir significativamente dichos profesionales, es en la optimización de personas motivadas y altamente efectivas en la organización, mediante su especialización en proyecto de vida, toma de decisiones y el desarrollo de procesos vocacionales los cuales posibiliten un mayor autoconocimiento, reconocimiento de valores, intereses, aptitudes, actitudes, habilidades, competencias, metas y expectativas que tienen los individuos, los cuales pueden contribuir al fortalecimiento personal y laboral. 


\section{Conclusiones}

A modo de conclusiones, se rescata la importancia de mantener personas colaboradoras motivadas dentro del sistema educativo, favorece el tener individuos autorrealizados, con mayor apertura a contribuir en el desarrollo de tareas y cumplimiento de los objetivos propuestos en la institución, ayudando en la obtención de productos de mayor calidad y con mayor eficacia. Todo ello es posible mediante una gestión institucional que en forma conjunta con el departamento de orientación logren articular líneas de trabajo conducentes al cumplimiento de lo propuesto.

La educación es un proceso dinámico, integral y complejo, el cual demanda de personas con una clara vocación y que se sientan motivadas en el desempeño de sus labores; campo en el cual la gestión institucional tiene un papel estratégico. Los profesionales en orientación, tienen las herramientas por su formación para colaborar en este proceso, mediante el trabajo conjunto con la dirección institucional, en aspectos como la comunicación, el proyecto de vida, las relaciones interpersonales, entre otros, los cuales beneficien a la persona y a su vez, a la organización como tal.

Existen factores internos y externos los cuales afectan a las personas colaboradoras dentro de los centros educativos; sin embargo, si se trabaja conjuntamente desde la dirección de la institución, en políticas que propicien el autocuidado de las personas colaboradoras, se pueden prevenir muchos factores disociadores que disminuirían el grado de motivación en los individuos. El papel de la administración de la educación en las organizaciones educativas es fundamental en este sentido.

Se debe rescatar que las personas colaboradoras de los centros educativos son seres integrales con intereses y necesidades propias, los cuales se deben reconocer, así como apoyar y fortalecer en busca de mantener una visión clara de cuáles son los propósitos de los individuos y cómo estos pueden contribuir en la búsqueda permanente de estrategias desde la gestión capaces de fortalecer el quehacer diario y el desarrollo de ideas productivas en pro de un mejor ambiente organizacional, armonioso, visionario y capaz de sobreponerse a los retos de la globalización.

Los profesionales y las profesionales de orientación con el apoyo de la gestión institucional pueden contribuir mediante el desarrollo de estrategias y planes de intervención, en la búsqueda de mayor calidad de vida en las personas trabajadoras, lo que a su vez puede favorecer a que la institución educativa se vea fortalecida y beneficiada mediante la tenencia de colaboradores y colaboradoras más comprometidos con la tarea, la misión y visión, base fundamental de la identificación con los propósitos específicos tenidos, los cuales benefician los procesos de enseñanza aprendizaje. 
La motivación en los individuos puede ser reforzada con la ayuda oportuna de profesionales de orientación, quienes mediante procesos de intervención individuales y colectivos, así como de educación integral, favorezcan a desarrollar competencias y habilidades que le posibiliten a las personas una mayor calidad de vida y por ende, alcanzar la autorrealización, aspecto intrínseco del cumplimiento de todas las tareas realizadas. Todo ello es posible en las organizaciones educativas con adecuados procesos de gestión institucional.

\section{Referencias}

Abarca, N. (1995). Psicología de la motivación. San José, Costa Rica: EUNED.

Aguilar, J. y Vargas, J. (2010). Comunicación asertiva. Network de Psicología. Recuperado en: http://www.conductitlan.net/psicologia_organizacional/comunicacion_asertiva.pdf

Alles, M. (2011). Desarrollo del talento humano basado en competencias. Buenos Aires: Granica.

Arce, J. (2000). Autoestima y proyecto de vida en los y las jóvenes. San José, Costa Rica: Izcandé.

Barquero, A. (2005). Administración de recursos humanos. San José, Costa Rica: EUNED.

Barrionuevo, L. (2001). Motivación para el trabajo en equipo. San José, Costa Rica: Editorial Norma.

Bisquerra, R. (1990). Orientación psicopedagógica para la prevención y el desarrollo. Barcelona: Boixareu.

Chacón, M. y Villalobos, D. (2005). Trabajo adolescente: repercusiones en la construcción del proyecto de vida de los adolescentes de la comunidad de concepción de Naranjo. (Tesis de licenciatura). Universidad de Costa Rica. San José, Costa Rica.

Condorí, F. (2003). El proceso de motivación en la empresa cooperativa. Matadero Nacional Montecillos R. L. Dirección Administrativa y Curtidos. (Tesis de licenciatura). Universidad Nacional. Heredia, Costa Rica.

División de Educación para el Trabajo. (2012). Plan de estudios de la carrera de orientación. Universidad Nacional. Heredia, Costa Rica: UNA.

Gómez, G. y Pin, J. (1994). Dirigir es educar. Madrid, España: McGraw-Hill.

Hageman, G. (2002). Motivación. Manual de implementación. México: LIMUSA. 
Quintero, N., Africano, N. y Faría, E. (abril, 2008). Clima organizacional y desempeño laboral del personal en la empresa Vigilantes Asociados Costa Oriental del Lago. Revista NEGOTIUM / Ciencias Gerenciales, 3(9), 33-51. Recuperado de http://www.revistanegotium.org.ve/ pdf/9/Art2.pdf

Ramírez, R, Abreu, J. y Badii, M. (marzo, 2008). La motivación laboral, factor fundamental para el logro de objetivos organizacionales: Caso empresa manufacturera de tubería de acero. Daena: International Journal of Good Conscience, 3(1), 143-185. Recuperado de http:// www.spentamexico.org/v3-n1/3(1)\%20143-185.pdf

Reeve, J. (1994). Motivación y emoción. Madrid: Editorial McGraw-Hill.

Sánchez, l.; Oliva, A. y Parra, Á. (2006). Empatía y conducta prosocial durante la adolescencia. Revista de Psicología Social, 21(3), pp. 259-271. Recuperado de http://personal.us.es/oliva/ prosocial.pdf

Solórzano, G. A. (2008). El hombre, un ser integral. Foro de Educación, 10, 347-367. Recuperado de http://www.forodeeducacion.com/numero10/021.pdf

Yañez, R., Arenas, M. y Ripoll, M. (2010). El impacto de las relaciones interpersonales en la satisfacción laboral general. Revista LIBERABIT, 16(2), 193-202. Recuperado de http://www. scielo.org.pe/pdf/liber/v16n2/a09v16n2.pdf 